\title{
Adaptive optics multiphoton microscopy
}

\author{
Emilio J. Gualda, Juan M. Bueno*, Pablo Artal \\ Laboratorio de Óptica, Universidad de Murcia, \\ Campus de Espinardo, Edificio CiOyN, 30100 Murcia, Spain \\ *bueno(o) um.es
}

\begin{abstract}
We have developed an adaptive optics multiphoton microscope. The multiphoton imaging system combines an ultrafast high-power laser, a scanning unit, a motorized Z-scan device and a photon-counting detector. The adaptive optics module is composed of a Hartmann-Shack wavefront sensor and a MEMS deformable mirror. The impact of compensating the aberrations of the laser beam is shown in a number of biological and non-biological samples. As examples, nonlinear fluorescence and second harmonic generation images of non-stained ex-vivo ocular tissues are compared with and without adaptive optics. The correction of the beam's aberrations increases both contrast and resolution in the non-linear microscope images.
\end{abstract}

Keywords: Multiphoton microscopy, adaptive optics, aberrations, ocular tissues.

\section{INTRODUCTION}

In the last years, the combination of imaging microscopy techniques and ultrafast lasers to produce non-linear signals has opened new opportunities of research in Biomedicine. Among the new methods for the visualization of smaller structures, non-linear imaging techniques are particularly interesting, such as Two-photon Excitation Fluorescence (TPEF) and Second Harmonic Generation (SHG) ${ }^{1,2}$. The aim of these techniques is to obtain selective information of the molecular composition of biological and non-biological samples while increasing the lateral and axial resolution and reducing the collateral damage. Both imaging techniques can be implemented in the same microscope obtaining complementary information. TPEF relies on non-linear absorption followed by fluorescence emission and is mainly used to visualize endogenous or exogenous fluorophores. SHG is based on non-linear scattering in molecules with inversion symmetry, such as collagen, and allows observing some morphological features without the need of staining procedures.

Despite the different nature of the processes, they share common advantages due to its non-linear behaviour. First, the use of infrared light as excitation allows to image deep into the sample avoiding ultraviolet excitation induced damage. Second, the reduction of the affected area to the focal volume reduces the out-of-focus photodamage and photobleaching. Finally, the intrinsic autoconfocality simplifies the setup, compared with confocal microscopes, avoiding the need of a pinhole in front of the detector and making easier the three-dimensional reconstruction of the sample under analysis, by combining the information obtained at different depths.

All these advantages make non-linear techniques a suitable platform for biological and medical research. Previous research efforts concentrated on optimizing the generated non-linear signal and reducing photobleaching effects by analyzing the phase-shape ${ }^{3}$, the temporal ${ }^{4}$ and the spectral ${ }^{5}$ properties of the laser source. However, little attention has been paid to the influence of the laser beam wavefront aberration (WA) on non-linear microscopy imaging. Similarly to linear microscopy, the image quality is first limited by the beam's aberrations. Other degradation sources include the optical elements of the imaging system (i.e. mirrors, lenses, microscope objective,...) and the specimen itself when indepth imaging is intended. These are locally different and depend on the morphology and composition of each biological sample. Aberrations increase the point-spread function (PSF) volume and, consequently, produce a reduction of axial and lateral resolution, contrast and the efficiency of the fluorescent processes ${ }^{6}$. Since within thick biological samples the penetration depth is reduced, an increase of the excitation power is needed what may produce sample damage.

Different approaches have been addressed to compensate for the in-depth specimen WAs, most of them without measuring directly the actual WA, combining genetic algorithms ${ }^{7}$ and adaptive optics $(\Lambda O)$ devices ${ }^{8}$. Others used complex systems to compute the WA's using interferometric ${ }^{9}$ techniques. In this work we show how non-linear microscopy images are significantly improved when an $A O$ device is used to compensate for the beam's aberrations.

MEMS Adaptive Optics IV, edited by S. Olivier, Thomas G. Bifano, Joel A. Kubby, Proc. of SPIE Vol. 7595 $75950 \mathrm{G} \cdot$ (c) $2010 \mathrm{SPIE} \cdot$ doi: 10.1117/12.845981 


\section{METHODS}

\subsection{Experimental setup}

The experimental system combining a multiphoton microscope and an AO module is shown in Figure 1. A femtosecond Ti:Sapphire laser $(760 \mathrm{~nm})$ was used as illumination source. This beam passes the $\mathrm{AO}$ module before entering the microscope. The AO module is composed of a Hartmann-Shack (IIS) wavefront sensor and a deformable mirror (DM) used respectively to measure and correct for the WAs. The IIS sensor sampled the wavefront with an array of microlenses $(0.15 \mathrm{~mm}$ pitch and $3.7 \mathrm{~mm}$ focal length). The DM was a gold coated MEMS-type (Boston Micromachines) with 140 actuators. A set of two galvanometric mirrors (M1 and M2) scanned the sample in the XY plane and a 7-scan motor moved the microscope objective to record stacks of images at different depths within the sample. The backscattered signals (both SIG and TPEF) from the sample passed a dichroic mirror (DIM) and the corresponding filter (F), to reach the detection unit (PMT). The control of image recording, as well as post-processing, was made via custom software. Images of the different samples were captured with and without AO.

The laser beam WA's were estimated from the recorded IIS images ${ }^{10}$ and expressed as a Zernike polynomial expansion up to $5^{\text {th }}$ order over a $3-\mathrm{mm}$ pupil. From each WA, the corresponding PSF (i.e. the far-field intensity distribution) was calculated. As image quality parameters, we used the root-mean-square (RMS) of the aberrations and the Strehl ratio. The appropriate mirror shape to compensate for the measured aberrations was determined in closed-loop.

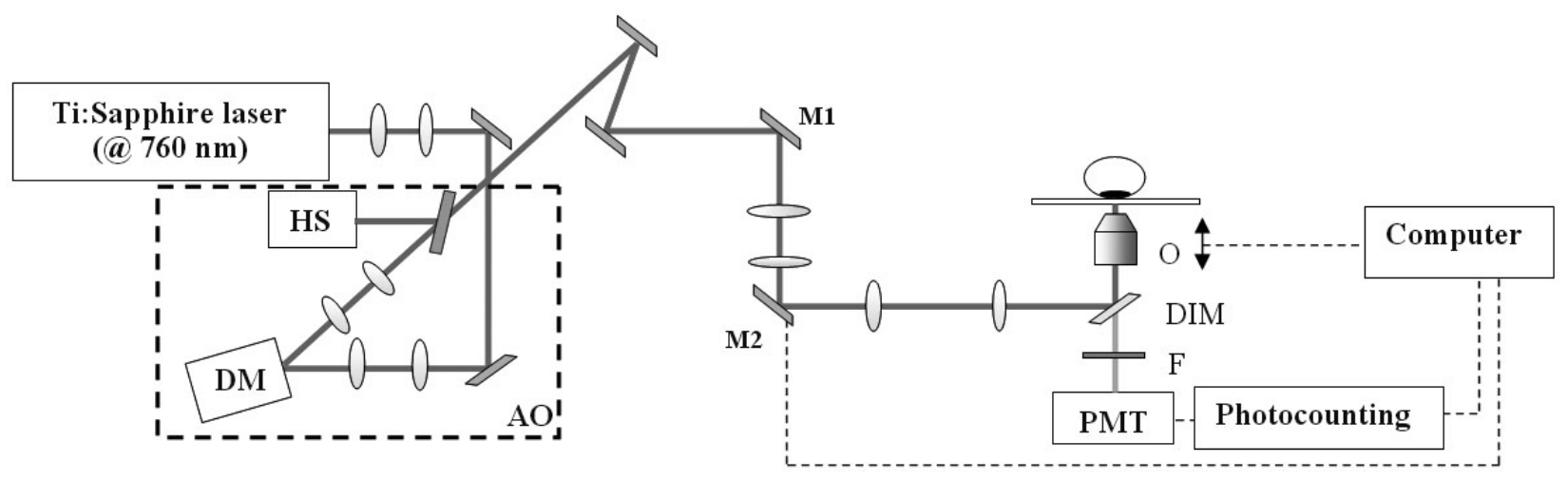

Figure 1. Adaptive optics multiphoton microscope. IIS, Hartmann-Shack wavefront sensor; DM, deformable mirror; M1 and M2, scanning mirrors; DIM, dichroic mirror; O, microscope objective; F, TPEF or SIIG filter; PMT, photomultiplier.

\subsection{Samples}

Both non biological and biological samples were used in the experiments reported here. The former included a piece of paper stained with a fluorescent marker which provided a strong TPEF signal. As biological samples, we used nonstained corneal and retinal tissues from human donors.

\section{RESULTS}

To illustrate the impact of aberrations on the image quality, we first studied the effects of astigmatism on imaging corneal collagen and the retinal nerve fiber layer. Results are shown in Figure 2. Whereas the stroma is made of collagen and provides SIG signal ${ }^{11,12}$, the retinal nerve fibers are autofluorescent ${ }^{13}$. Inducing an amount of astigmatism of 0.5 diopters noticeably reduces the signal levels in the images. In particular, a reduction of $28 \%$ and $73 \%$ in SHG and TPEF signals respectively occur. Moreover, the images recorded with astigmatism also present an overall lower image quality.

Figure 3 shows the compares the WAs and their associated PSF with and without adaptive optics. After correction the root-mean-square error of the WA was reduced from 0.34 to 0.06 microns (Strehl ratio increased from 0.19 to 0.84 ). Figure 4 depicts the corresponding individual Zernike coefficients (up to $4^{\text {th }}$ order) of these WAs. 

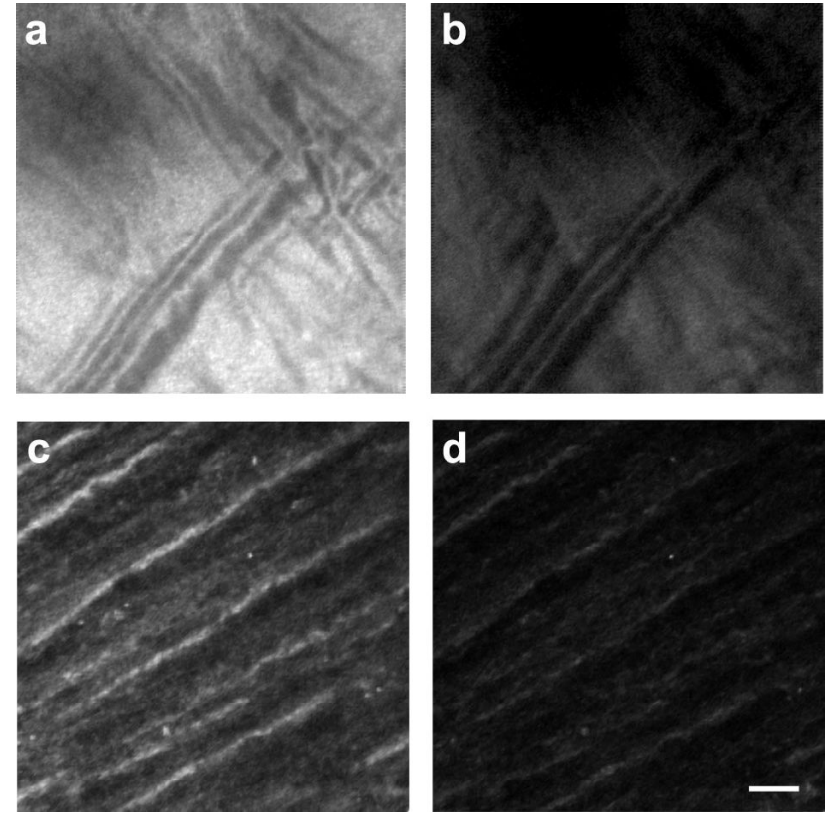

Figure 2. Effect of astigmatism on the image quality of SHG (a and b) and TPEF (c and d) images of the cornea and retinal nerve fiber layer from ex-vivo human eyes. (a and c) Image control; (b) -0.5 diopters of $45^{\circ}$ astigmatism; (d) -0.5 diopters of vertical astigmatism. Bar length: $25 \mu \mathrm{m}$. Pairs of images share the gray scale.

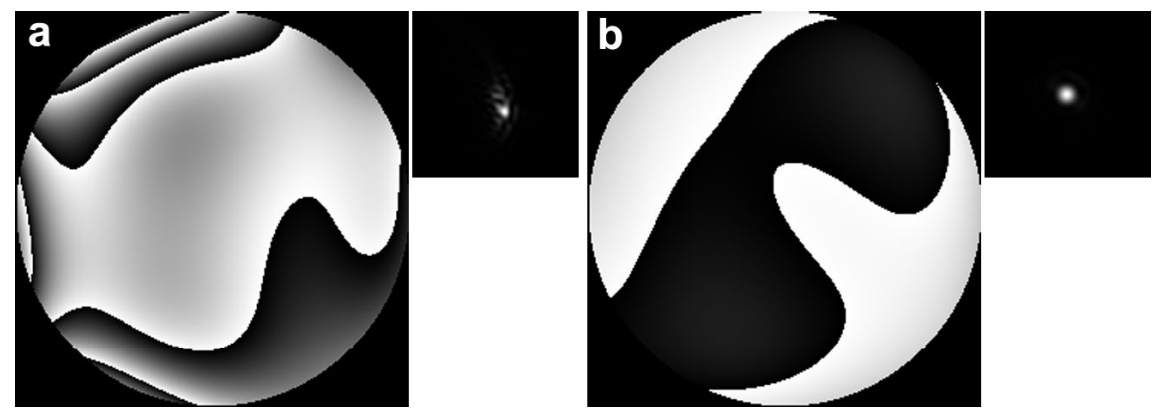

Figure 3. Laser beam WAs (2 $\pi$-phasewrapped) and associated PSFs before (a) and after using AO correction (b).

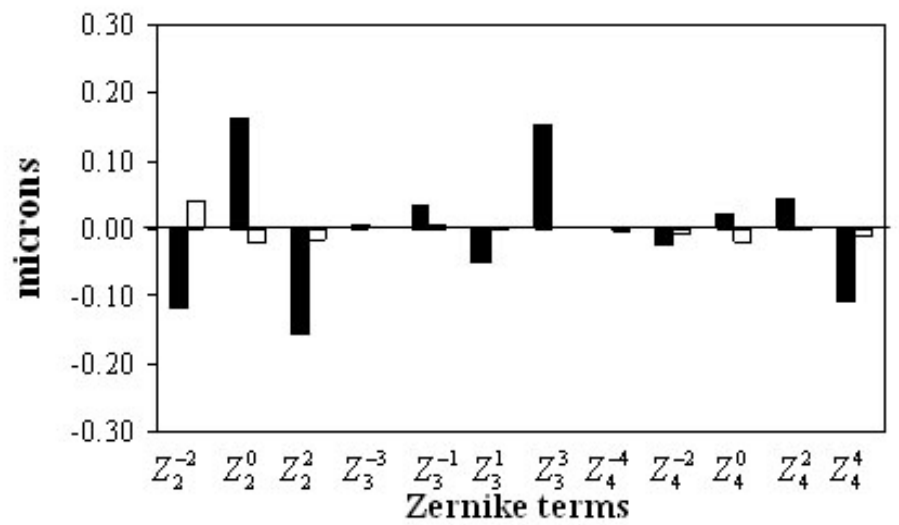

Figure 4. Individual 7ernike terms (up to $4^{\text {th }}$ order) of laser beam WA before (black bars) and after (white bars) using AO correction.

The effect of aberration correction in TPEF microscopy is depicted in Figure 5 for a stained piece of paper. The uncorrected as well as corrected cases are shown. The benefit of using AO for TPEF images is clearly seen in this nonbiological sample. An increase in the total signal intensity of around 50\% was reached. Moreover, more spatial details are observed in the corrected TPEF image. 

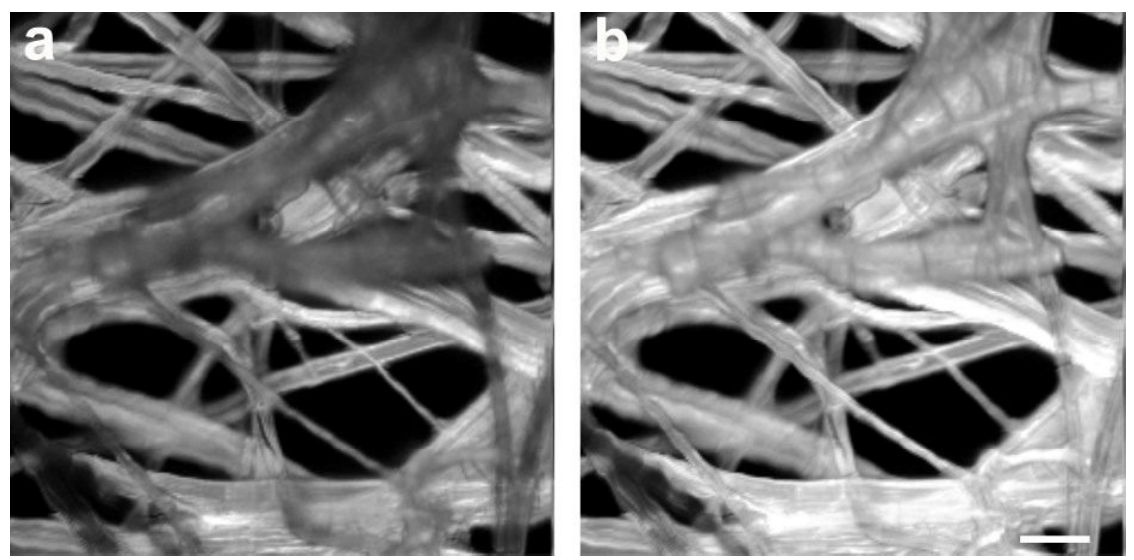

Figure 5. Effect of using AO to compensate for the laser beam WAs on TPEF imaging. The sample corresponds to a piece of

fluorescent paper. (a) Adaptive optics OFF; (b) Adaptive optics ON. Gray levels represent the same intensity for both images. Bar size: 50 microns.

In the following we show two examples of improvement in TPEF microscopy imaging for ocular tissues. In a section of a human retina Figure 6 shows the aberration-corrected TPEF image to be compared with the image of the sample without aberration correction. The total intensity increased almost three-fold in the image recorded with the corrected beam. In Figure 7 presents the mosaic of photoreceptors in a porcine retina with and without AO. For the later the total intensity in the image was almost doubled with respect to the case when $\mathrm{AO}$ was not in operation. For both examples, the contrast and resolution in the image with the $\mathrm{AO}$ activated were enhanced and retinal features were better visualized.
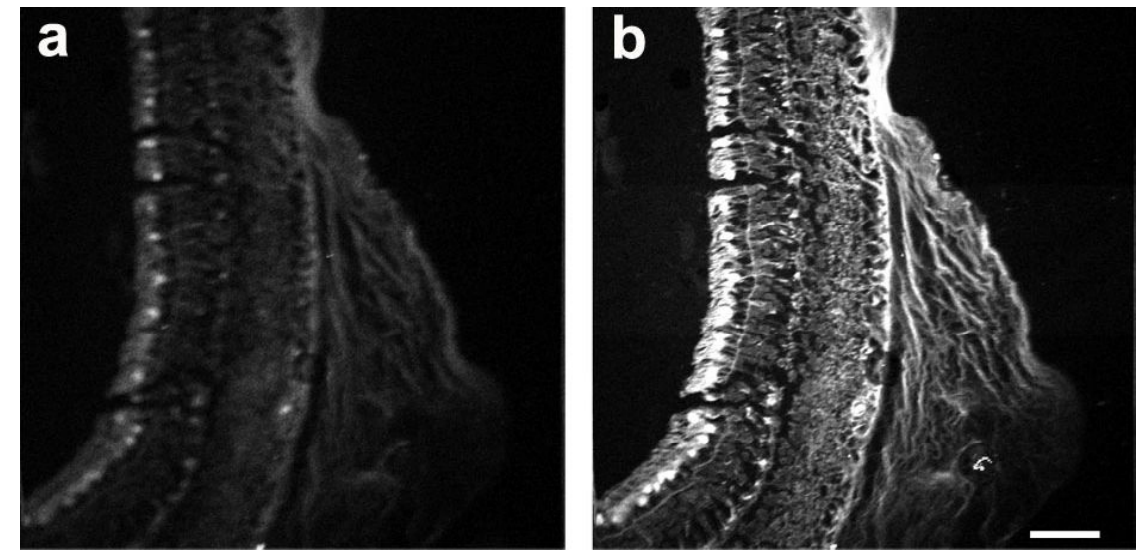

Figure 6. TPEF microscopy images of a histological section of an ex-vivo human retina. The visibility of small retinal features after correcting for the laser beam aberrations is improved. Images share the same grey scale. Bar size: 50 microns.
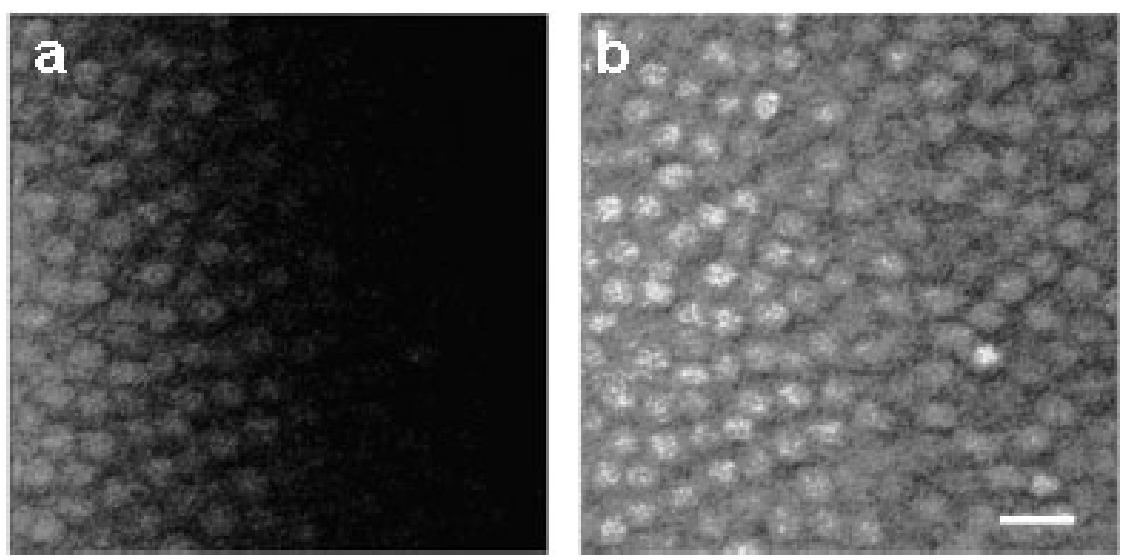

Figure 7. TPEF microscopy images of the mosaic of photoreceptors in an ex-vivo porcine. The visibility of individual photoreceptors is clearly improved when using AO. Images share the same grey scale. Bar size: 15 microns. 


\section{CONCLUSIONS}

We have developed a system combining a multiphoton microscope with $\mathrm{AO}$ in order to improve the quality of non-linear microscopy images. As a first step, the influence of lower order aberration was reported when imaging human corneal and retinal tissues. Then we presented TPEF and SIIG images of both biological and non-biological samples with and without compensation of the aberrations of the laser beam. The MEMS deformable mirror was able to reduce the amount of aberrations to nearly negligible levels. We showed that by improving the beam quality, both the contrast and resolution in TPEF images of non-stained retinal tissues increases. This approach also reduced the required excitation power, minimizing the phototoxicity side-effects within the imaged sample. In particular, this benefit may be important in the future diagnosis of ocular pathologies or in the improvement of laser ablation surgery techniques. Further experiments with the adaptive optics microscope will be carried out in the near future to pre-compensate for the aberrations produced by the sample itself.

\section{ACKNOWLEDGEMENTS}

This research was supported in part by the "Ministerio de Educación y Ciencia", Spain (grant FIS2007-64765), Fundación Séneca, Murcia, Spain (04524/GERM/06) and CONSOLIDER-INGENIO 2010 (CSD2007-00033). Ocular tissues were kindly provided by the "Servicio de Oftalmología of the Hospital Virgen de la Arrixaca", Murcia, Spain.

\section{REFERENCES}

1. Denk, W., Strickler, J. H. and Webb, W. W., "Two-photon laser scanning fluorescence microscopy," Science 248, 73-76 (1990).

2. Guo, Y, Ho, P. P., Savage, II., Harris, D., Sacks, P., Schantz, S., Liu, F., Zhadin, N. and Alfano, R. R. "Secondharmonic tomography of tissues," Opt. Lett. 22, 1323-1325 (1997).

3. Pastirk, I., Dela Cruz, J. M., Walowicz, K. A., Lozovoy, V. V. and Dantus M. "Selective two-photon microscopy with shaped femtosecond pulses," Opt. Express, 11, 1695-1701 (2003).

4. Tang, S., Krasieva, T. B., Chen, Z., Tempea, G. and Tromberg, B. J. "Effect of pulse duration on two-photon excited fluorescence and second harmonic generation in nonlinear optical microscopy," J.Biomed. Opt. 11, 020501/1-020501/3 (2006).

5. $\mathrm{Xu}, \mathrm{C}$. and Webb W. W. "Measurement of two-photon excitation cross sections of molecular fluorophores with data from 690 to 1050 nm," J. Opt. Soc. Am. B 13, 481-491 (1996).

6. Bueno, J. M., Vohnsen, B., Roso L. and Artal, P., "Temporal wavefront stability of an ultrafast high-power laser beam," Appl. Opt. 48, 770-777 (2009).

7. Sherman, L., Ye, J. Y., Albert, $O$. and Norris, T. B. "Adaptive correction of depth-induced aberrations in multiphoton scanning microscopy using a deformable mirror," J. Micros. 206, 65-71(2002).

8. Neil, M. A. A., Jusï Kaitis, R., Booth, M. J., Wilson, T., Tanaka, T. and Kawata, S. "Adaptive aberration correction in a two-photon microscope," J. Micros. 200, 105-108 (2000).

9. Schwertner, M., Booth, M. J. and Wilson, T. "Characterizing specimen induced aberrations for high NA adaptive optical microscopy," Opt. Express 12, 6540-6552 (2004).

10. Prieto P. M., Vargas-Martín F., Goelz S., Artal P., "Analysis of the performance of the Hartmann-Shack sensor in the human eye", J. Opt. Soc. Am. A 17, 1388-1398 (2000).

11. Yeh, A. T., Nassif, N., Zoumi, A. and Tromberg, B.J., "Selective corneal imaging using combined second-harmonic generation and two-photon excited fluorescence," Opt. Lett. 27, 2082-2084 (2002).

12. Morishige, N., Petroll, W. M., Nishida, T., Kenney, M. C. and Jester, J.V., "Noninvasive corneal stromal collagen imaging using two-photon-generated second-harmonic signals," J. Cataract. Refract. Surg. 32, 1784-1791 (2006).

13. Gualda, E. J., Bueno J. M. and Artal, P., "Wavefront optimized non-linear microscopy of ex-vivo human retinas," J. Biom. Opt. (submitted). 